\title{
Development of Canadian Recommendations for the Management of ANCA-Associated Vasculitides: Results of the National Needs Assessment Questionnaire
}

\author{
Leilani Famorca ${ }^{1}$, Marinka Twilt ${ }^{2}$, Lillian Barra ${ }^{3}$, Volodko Bakowsky ${ }^{4}$, Susanne Benseler ${ }^{5}$, David Cabral ${ }^{6}$, \\ Simon Carette ${ }^{7}$, Navjot Dhindsa ${ }^{8}$, Aurore Fifi-Mah ${ }^{9}$, Michelle Goulet ${ }^{10}$, Nader Khalidi ${ }^{11}$, Majed Khraishi ${ }^{12}$, \\ Lucy McGeoch $^{7}$, Nataliya Milman ${ }^{13}$, Christian Pineau ${ }^{14}$, Kam Shojania ${ }^{15}$, Regina Taylor-Gjevre ${ }^{16}$, \\ Tanveer Towheed ${ }^{17}$, Judith Trudeau ${ }^{18}$, Elaine Yacyshyn ${ }^{19}$, Patrick Liang ${ }^{20}$ and Christian Pagnoux ${ }^{*}, 7$ \\ for the Canadian Vasculitis network (CanVasc) ${ }^{\S}$
}

\begin{abstract}
${ }^{1}$ Langs Community Centre, Cambridge, Ontario, Canada; ${ }^{2}$ Division of Rheumatology, Department of Pediatrics, The Hospital for Sick Children, Toronto, Ontario, Canada, ${ }^{3}$ Division of Rheumatology, St. Joseph's Health Care, London, Ontario, Canada; ${ }^{4}$ Division of Rheumatology, Nova Scotia Rehabilitation Center, QEII HSC, Halifax, Nova Scotia, Canada; ${ }^{5}$ Division of Rheumatology, Department of Paediatrics, Alberta Children's Hospital, Calgary, Alberta, Canada; ${ }^{6}$ Division of Rheumatology, BC Children's Hospital, Vancouver, British Columbia, Canada; ${ }^{7}$ Department of Rheumatology, Mount Sinai Hospital, Toronto, University of Toronto, Ontario, Canada; ${ }^{8}$ Section of Rheumatology, University of Manitoba, Arthritis Centre, Winnipeg, Manitoba, Canada; ${ }^{9}$ Division Division of Rheumatology, South Health Campus, University of Calgary, Calgary, Alberta, Canada; ${ }^{10}$ Division of Internal Medicine, Hôpital du SacréCoeur de Montréal, Montréal, Quebec, Canada; ${ }^{11}$ Division of Rheumatology, St. Joseph's Healthcare Hamilton, McMaster University Hamilton, Ontario, Canada; ${ }^{12}$ Division of Rheumatology, Memorial University of Newfoundland, St. John's, Newfoundland, Canada; ${ }^{13}$ Arthritis Centre at the Ottawa Hospital, Riverside Campus, Ottawa, Ontario, Canada; ${ }^{14}$ McGill University, MUHC Lupus and Vasculitis clinic, Montréal, Québec, Canada; ${ }^{15}$ Division of Rheumatology, Vancouver General Hospital and St. Paul's Hospital, Vancouver, British Columbia, Canada; ${ }^{16}$ Division of Rheumatology, Department of Medicine, Royal University Hospital, University of Saskatchewan, Saskatoon, Canada; ${ }^{17}$ Department of Medicine, Queen's University, Kingston, Ontario, Canada; ${ }^{18}$ Division of Rheumatology, CHAU de Lévis, Lévis, Quebec, Canada; ${ }^{19}$ Division of Rheumatology, University of Alberta, Edmonton, Alberta, Canada;

${ }^{20}$ Division of Rheumatology, Centre Hospitalier Universitaire de Sherbrooke, Sherbrooke, Quebec, Canada
\end{abstract}

\begin{abstract}
Objectives: To study variations in Canadian clinical practice patterns for the management of ANCA-associated vasculitis (AAV) and identify points to consider for the development of national recommendations.

Material and Methodology: A 30-item needs assessment questionnaire was sent to all members of the Canadian Vasculitis network (CanVasc), Canadian Rheumatology Association (CRA), Canadian Thoracic Society (CTS) and Canadian Society of Nephrology (CSN). Respondent characteristics, practice patterns, concerns and expectations were analyzed.

Results: Among 132 physicians who followed at least 1 vasculitis patient and responded to the survey, 39\% stated that they felt confident in their management of AAV. Several variations in practice were observed regarding diagnostic procedure, induction and maintenance treatments and use of biologics; some were due to logistic constraints (difficulties in access to some specific tests, drugs or care; lack of health care coverage for the costs). The top 5 topics for which recommendations are expected involve treatment for remission induction, maintenance, refractory disease, and relapse as well as biologics.
\end{abstract}

Conclusion: Practice variations identified in this needs assessment survey will serve to formulate key questions for the development of CanVasc recommendations.

Keywords: ANCA-associated vasculitis, CanVasc, physician practice patterns.

*Address correspondence to this author at the Mount Sinai Hospital, The Joseph and Wolfe Lebovic Building, R 2-220, 60 Murray Street, Toronto, ON, M5T 3L9, Canada; Tel: +1-416-586-4800, Ext. 8549; Fax: +1-416586-8766; E-mail: cpagnoux@mtsinai.on.ca

${ }^{\S} \mathrm{A}$ complete and updated list of CanVasc founders, bureau, core, associated and affiliated members can be downloaded from: http://www.canvasc.ca/Inf ormationCanVasc.htm

\section{INTRODUCTION}

Antineutrophil cytoplasm antibody (ANCA)-associated vasculitis (AAV) refers to granulomatosis with polyangiitis (GPA, formerly Wegener's granulomatosis), microscopic polyangiitis (MPA) and eosinophilic granulomatosis with polyangiitis (formerly Churg-Strauss syndrome) [1]. Combined estimates of prevalence and incidence of these 3 
systemic diseases are 56-273 per million population and 339 per million population per year, respectively, according to studies conducted in North America, Europe and Asia [2, 3]. Frequencies of AAV in Canada are likely to be comparable [4]. This rarity limits both the personal and general clinical experiences with such patients and potentially makes their diagnosis and treatment challenging. All 3 diseases are potentially life-threatening, and tissue or organ damage from the disease or treatment is common in survivors. Hence, clinical practice guidelines can be useful for synthesizing and transmitting evidence-based management strategies to appropriate users.

The Canadian Vasculitis research network (CanVasc) was created in November 2010 and gathered several physicians across Canada with experience and interest in vasculitis. The ultimate objective of this group is to improve the diagnosis, management and outcomes of vasculitis in Canadian patients. The stated aims of CanVasc include the initiation and development of Canadian-specific recommendations for AAV clinical management, taking into account cultural and healthcare variations in Canada, and incorporating results of recent randomised controlled trials $[5,6]$. As recommended and illustrated with other disorders [7], an initial needs assessment questionnaire was distributed to Canadian healthcare professionals from different medical specialties involved in vasculitis management. Input was sought regarding current knowledge, knowledge gaps, uncertainties and challenges in the diagnostic, therapeutic and follow-up management of AAV in Canada. The specific objectives were to formulate a series of relevant clinical questions to direct the literature search and guide the development of practice recommendations.

\section{MATERIALS AND METHODOLOGY}

A questionnaire asking about demographic/practice characteristics and including general questions on diagnostic investigations, treatments, follow-up practice for AAV patients and gaps in knowledge about the management of these patients was developed by CanVasc core members (primarily by L.F., M.T., C.Pa. and P.L. - available as an online Supplementary material). The questionnaire was submitted for review to the Canadian Rheumatology Association (CRA) Therapeutics Committee, Canadian Thoracic Society (CTS) and Canadian Nephrology Society (CSN). The questionnaire was developed by use of Survey Monkey (www.surveymonkey.com) and a pilot version was tested in Spring 2012. The questionnaire was distributed in Summer 2012 to all members of the CRA anonymously. A link inviting CTS and CNS members to complete the survey was also added to their respective newsletters for dissemination.

The survey remained open for 3 months, with one reminder email sent to CanVasc and CRA members as well as CSN and CTS administrative offices 1 month before survey closure on October 15, 2012. The collected data were then extracted to Microsoft Excel ${ }^{\circledR}$ for descriptive analysis.

\section{RESULTS}

In all, 136 members of the CRA, CTS and/or CanVasc responded to the survey, and 132 completed it. Four respondents were not invited to go beyond the first question because they reported not seeing any vasculitis patients. The mean age of the respondents was $48 \pm 11$ years and $62 \%$ had been in practice for $>10$ years. About half $(52 \%)$ were male and the greatest proportion of respondents resided in Ontario $(n=54)$, followed by Québec $(n=25)$, then the western provinces (British Columbia $\mathrm{n}=13$; Alberta, $\mathrm{n}=18$; Saskatchewan $\mathrm{n}=3$; Manitoba, $\mathrm{n}=2$ ) and eastern provinces (New Brunswick, $n=5$; Nova Scotia, $n=4)(8$ did not respond to the question about their work location). A total of $64 \%$ of respondents reported practicing in academic or teaching hospitals. Respondents in order of frequency were rheumatologists (adult, 63\%; pediatric, 6\%), respirologists $(23 \%)$, internists $(4 \%)$ and primary care physicians $(1.5 \%)$. A small insert with a link to the survey was provided in the CSN newsletter, but no nephrologists completed the online questionnaire.

Overall, $77 \%$ of respondents reported seeing 3 cases of GPA/MPA or more per year, and 33\% reported seeing 3 cases of EGPA or more per year in their center (24 respondents reported being members of a vasculitis network such as CanVasc or A Registry for Children with Vasculitis: e-entry [ARCHiVe]); 77 of 108 respondents (71\%) not selfdeclared as members of a vasculitis network reported managing AAV in patients themselves without "referring them to a referral center for vasculitis at least once to be enrolled in a cohort study". A total of 15 respondents (11\%) reported that their center had some local written protocol for treatment of AAV.

As shown in Table 1, most diagnostic investigations were ordered homogeneously by most physicians, including complete blood cell count, renal parameters, C-reactive protein level and/or sedimentation rate (both ordered systematically by $>85 \%$ of the respondents), as well as testing for ANCA (ordered systematically by $91 \%$ of the respondents, with 2 respondents reporting that the cost of the test for patients was prohibitive). Testing for complement fractions ( $\mathrm{C} 3$ and $\mathrm{C} 4)$, serum protein electrophoresis and screening for lupus anticoagulant were ordered systematically by $76 \%, 47 \%$ and $42 \%$ of respondents, respectively, during diagnostic work-up. Screening for antiglomerular basement membrane (anti-GBM) antibodies, voluntarily not included in the list of possible diagnostic investigations, was added in the text box for "other tests/comments" by 3 respondents. Chest plain X-ray was ordered systematically by most physicians $(96 \%)$, tuberculin skin test by $25 \%$ and some CT scan of organs involved by $57 \%$ of respondents.

For remission induction, almost all respondents reported that they regularly used corticosteroids, intravenous (IV) or oral cyclophosphamide. A total of $68 \%$ and $64 \%$ reported having used rituximab or methotrexate for induction; $33 \%$ reported that they had used mycophenolate mofetil and 7\% an anti-tumor necrosis factor alpha agent for induction. Only $48 \%$ reported having used plasma exchange as part of induction therapy. Two respondents commented on the sequential use of IV then oral cyclophosphamide, and 3 on practical difficulties in setting up IV cyclophosphamide infusions. The mean duration of induction was $\leq 6$ months for $88 \%$ of respondents. One question asked about the use of 
Table 1. Main results from the needs assessment questionnaire on practice patterns for management of ANCA-associated vasculitis (number of respondents seeing at least 1 vasculitis patient $=132$ ).

\begin{tabular}{|c|c|c|c|}
\hline \multirow[t]{2}{*}{ Questionnaire Items } & \multicolumn{2}{|c|}{ Answers* } & \multirow[t]{2}{*}{ Comments } \\
\hline & Always & Never & \\
\hline \multicolumn{4}{|l|}{ Diagnostic investigations } \\
\hline Complete blood count & $100 \%$ & 0 & \\
\hline Liver function test & $94 \%$ & 0 & \\
\hline Renal function test & $100 \%$ & 0 & \\
\hline C-reactive protein & $88 \%$ & $1 \%$ & \\
\hline Von Willebrand factor antigen & $7 \%$ & $58 \%$ & \\
\hline Complement $\mathrm{C} 3 / \mathrm{C} 4$ & $76 \%$ & $2 \%$ & \\
\hline ANCA & $91 \%$ & $6 \%$ & "Some refuse ANCA testing because of the cost" \\
\hline Antinuclear autoantibody & $85 \%$ & 0 & \\
\hline Serum protein electrophoresis & $47 \%$ & $11 \%$ & \\
\hline Lupus anticoagulant and/or anticardiolipin & $42 \%$ & $6 \%$ & \\
\hline Which other investigations for patients with suspected AAV? & & & "Depends on clinical presentation" \\
\hline CT scan /MRI of involved organ & $57 \%$ & 0 & \\
\hline Echocardiogram & $30 \%$ & $2 \%$ & \\
\hline Cardiac MRI (EGPA) & $7 \%$ & $31 \%$ & \\
\hline IgE level (EGPA) & $52 \%$ & $4 \%$ & \\
\hline Electromyogram & $4 \%$ & $11 \%$ & \\
\hline \multicolumn{4}{|l|}{ Induction treatments } \\
\hline \multicolumn{4}{|l|}{ Which of the following treatments do you use to induce remission? } \\
\hline Corticosteroids (intravenous pulse) & $94 \%$ & $6 \%$ & \\
\hline Oral corticosteroids & $94 \%$ & $0 \%$ & \\
\hline Oral cyclophosphamide & $87 \%$ & $13 \%$ & \\
\hline Intravenous cyclophosphamide & $91 \%$ & $9 \%$ & $\begin{array}{l}\text { "IV cyclophosphamide not infused at my centre", } \\
\text { "initial IV cyclophosphamide pulse then switch to daily oral" }\end{array}$ \\
\hline Methotrexate & $64 \%$ & $36 \%$ & "methotrexate and azathioprine for mild/limited disease" \\
\hline Azathioprine & $47 \%$ & $53 \%$ & \\
\hline \multicolumn{4}{|l|}{ Maintenance treatment } \\
\hline Rituximab & $32 \%$ & $68 \%$ & "would use rituximab if could get it before relapse" \\
\hline Infliximab & $3 \%$ & $97 \%$ & \\
\hline Leflunomide & $17 \%$ & $83 \%$ & \\
\hline Mycophenolate mofetil & $53 \%$ & $47 \%$ & \\
\hline Trimethoprim / sulfamethoxazole $(160 / 800 \mathrm{mg})$ twice daily & $60 \%$ & $40 \%$ & \\
\hline \multicolumn{4}{|l|}{ Follow-up } \\
\hline \multicolumn{4}{|l|}{ Which of the following tests to monitor your patients? } \\
\hline Complete blood count & $100 \%$ & 0 & \\
\hline Liver function tests & $86 \%$ & $2 \%$ & \\
\hline Creatinine & $98 \%$ & 0 & \\
\hline ANCA & $57 \%$ & $7 \%$ & \\
\hline ESR & $79 \%$ & $1 \%$ & \\
\hline $\mathrm{C}$ - reactive protein & $84 \%$ & $3 \%$ & \\
\hline Urinalysis & $91 \%$ & $1 \%$ & \\
\hline CT scan of chest and/or sinus & $17 \%$ & $6 \%$ & \\
\hline Pulmonary function tests & $30 \%$ & $7 \%$ & \\
\hline Electromyogram & 0 & $41 \%$ & \\
\hline Bone mineral density & $49 \%$ & $6 \%$ & \\
\hline CD19+ B cell count (in patients who received rituximab) & $14 \%$ & $67 \%$ & \\
\hline Urine cytology (patients who received cyclophosphamide) & $43 \%$ & $22 \%$ & \\
\hline Cystoscopy (patients who received cyclophosphamide) & $9 \%$ & $33 \%$ & "cystoscopy only if persistent microscopic hematuria" \\
\hline
\end{tabular}

* Respondents could skip questions, or chose between Always / Sometimes / Never.

$\dagger$ At the time the survey was conducted, rituximab was not yet approved in all Canadian provinces for induction in adult patients with severe AAV (and certain other specific criteria that may vary according to each province).

AAV: ANCA-associated vasculitis; ANCA: anti-neutrophil cytoplasmic antibodies; EGPA: eosinophilic granulomatosis with polyangiitis; ESR: erythrocyte sedimentation rate. 
rituximab for first-line induction treatment, assuming that it was approved and covered without restriction: $72 \%$ of respondents stated that they would not use it systematically for all their newly diagnosed patients and would thus still use cyclophosphamide. In contrast, $80 \%$ would use rituximab systematically for all newly diagnosed women of childbearing age, and $89 \%$ would use it systematically to induce remission in relapsing patients, regardless of the previous dosing of cyclophosphamide they may have received. Prophylaxis against Pneumocystis jiroveci pneumoniae when using cyclophosphamide was prescribed systematically by $83 \%$ of respondents, and sometimes by $16 \%$. Gonadotropinreleasing hormone for women of child-bearing age, oocyte collection or sperm preservation when using cyclophosphamide had never been used or considered a possibility for $39 \%, 33 \%$ and $25 \%$ of respondents, respectively, mainly because of the difficulty in obtaining coverage for the drugs, non-availability of the technique locally, and/or acuteness of AAV precluding any delay in cytotoxic initiation.

For remission maintenance, azathioprine and methotrexate were the most commonly used agents. In all, $23 \%$ of the respondents also selected continuous oral cyclophosphamide as a possible maintenance drug, 53\% mycophenolate mofetil, and 32\% rituximab re-infusions. Before prescribing azathioprine, $24 \%$ of the prescribers systematically genotyped or measured thiopurine methyltransferase activity, and a further $17 \%$ reported they would do so if the test were available at their institution and/or if the patient did not have to pay for it. Planned and/or optimal duration of maintenance therapy was up to 18 months for $18 \%$ of the respondents and longer than 4 years for $22 \%$ ("as long as required", "as long as tolerated", "maybe forever"). Average duration of corticosteroid therapy was $\leq 6$ months for $10 \%$ of respondents and exceeded 2 years for $12 \%$.

For patient follow-up, in parallel with standard tests such as complete blood cell count or C-reactive protein level, $57 \%$ and $49 \%$ always ordered serial ANCA testing and bone mineral density testing, respectively. For past recipients of cyclophosphamide, repeat urine cytology was ordered systematically by $43 \%$ of the respondents and regular cystoscopy by $9 \%$.

Overall, 39\% of the respondents stated that they felt confident in their management of AAV in patients. Among the possible topics to be covered in recommendations for AAV management, those rated with highest priority were remission induction treatment (rated with highest priority for $47 \%$ of respondents), then treatment of refractory disease (for 29\%) and relapse (for 25\%), indication and use of biologics (for 24\%) and maintenance therapy (for 19\%). Diagnostic procedures, prevention and monitoring of drugrelated toxicity, disease monitoring, and treatments for specific age groups (children, older adults) were the highest priority for only $13 \%, 10 \%, 9 \%$, and $7 \%$ of respondents, respectively.

\section{DISCUSSION}

Through this needs assessment questionnaire completed by 132 Canadian healthcare professionals managing AAV, practice variations were observed regarding diagnostic workup, induction and maintenance treatments, use of biologics and patient follow-up. The identification of these variations across Canadian centers and the prioritization by the survey respondents of the topics to be covered are now being taken into account in the development of the CanVasc recommendations for the management of AAV.

This study is one of the first major collaborative and multidisciplinary efforts for vasculitis in Canada and included an extensive evaluation of all aspects of the management of AAV. However, the aim of this needs assessment questionnaire was not to extensively analyze and compare the practice patterns in Canada, as several limitations in our study precluded such analyses. The specific aim of the survey was to help identify all relevant topics that could warrant recommendations. There are no published guidelines to our knowledge for the development or reporting of the results of such a needs assessment questionnaire and no consensual definition of the threshold at which the variability in patient care would warrant actions to harmonize care, such as the development of recommendations. The interpretation of our findings and the observed practice variations must thus remain cautious and considered only as results of preliminary but required work, prior to the development of the recommendations.

Several specificities must be taken into account when considering access to care and therapeutic management of patients with these rare conditions in Canada. Although several referral centers for AAV have been identified to participate in CanVasc (http://www.canvasc.ca), mainly based on their recruitment and experience with $\mathrm{AAV}$, the population density of Canada is highly variable and widely distributed. The cost coverage and access to some examinations, drugs and/or studies can be problematic for patients in non-referral centers; geographic isolation from major referral centers in the vast Canadian land mass might be a significant barrier.

Other possible reasons for the practice variations observed might include the variable personal experience of the respondents with these rare diseases and their knowledge of vasculitis in general and new developments in the field. For example, we found it surprising (and, if true, concerning) that $23 \%$ of the respondents reported the use of continuous oral cyclophosphamide for maintenance therapy after induction (the question was "For remission maintenance therapy of ANCA-associated vasculitis, what medications do you use [usually in combination with prednisone]?").

However, we may have failed to identify all practice variations for AAV considered by Canadian physicians. Of note, no nephrologists answered the survey, likely because the survey was not advertised enough among them and they did simply not notice it. Our survey was concurrent with development of the Kidney disease/improving global outcomes (KDIGO) Clinical Practice Guideline for Glomerulonephritis, [8] developed around the same time as the survey, and may also have diverted some nephrologists' attention from this survey. However, several nephrologists are members of CanVasc and are involved in the ongoing development of the recommendations, and, although important, renal disease remains only one of the numerous facets of the multi-system AAV. General internists were also 
underrepresented among the respondents. However, in Canada, the primary care physicians who manage patients with vasculitis are more often rheumatologists, nephrologists and/or respirologists, as compared with several European countries in which general internists have a more central role. In addition, the survey questions were pilot-tested with a panel of CanVasc-affiliated rheumatologists, nephrologists and other specialists as well as internist experts and trainees who frequently co-manage AAV in patients.

Finally, the needs assessment questionnaire was conducted before rituximab was approved as induction therapy for adult patients with severe AAV in all Canadian provinces (official approval by Health Canada in December 2011, then first in Ontario in February 2012). However, we asked whether its approval would affect physicians' choice for induction treatment. Surprisingly, despite rituximab being approved only for induction treatment, almost onethird of the respondents reported its use for maintenance, as suggested by the results of a few recent series and studies [912], and likely covered by patients' own drug insurance plans.

Ultimately, reduced variation in practice could be achieved with the development, dissemination and acceptance of consensus recommendations. However, the impact on patient outcomes, before and after the dissemination of the recommendations, will need to be assessed.

\section{CONCLUSION}

Our needs assessment survey identified practice variations in Canada and the most important points to consider in the development of CanVasc recommendations for the management of AAV. Our article also provides a good example of the difficulties and limitations of such needs assessment questionnaires, for which there is at present, to our knowledge, no particular accepted method for development, interpretation or reporting.

\section{CONFLICT OF INTEREST}

We declare that there is no specific funding, financial contribution or conflict of interest to report in relation with the work being reported.

\section{ACKNOWLEDGMENTS}

Drs. Gerard P. Cox, Pearce G. Wilcox and the Canadian Thoracic Society (CTS); Dr. Shahin Jamal, the Canadian
Rheumatology Association (CRA) and CRA Therapeutic Committee members; Dr. Jim Barton and the Canadian Society of Nephrology.

\section{REFERENCES}

[1] Jennette JC, Falk RJ, Bacon PA, et al. 2012 revised International Chapel Hill Consensus Conference Nomenclature of Vasculitides. Arthritis Rheum 2013; 65: 1-11.

[2] Mohammad AJ, Jacobsson LT, Westman KW, Sturfelt G, Segelmark M. Incidence and survival rates in Wegener's granulomatosis, microscopic polyangiitis, Churg-Strauss syndrome and polyarteritis nodosa. Rheumatology (Oxford) 2009; 48: 15605.

[3] Gibelin A, Maldini C, Mahr A. Epidemiology and etiology of wegener granulomatosis, microscopic polyangiitis, churg-strauss syndrome and goodpasture syndrome: vasculitides with frequent lung involvement. Semin Respir Crit Care Med 2011; 32: 264-73.

[4] Anderson K, Klassen J, Stewart SA, Taylor-Gjevre RM. Does geographic location affect incidence of ANCA-associated renal vasculitis in northern Saskatchewan, Canada? Rheumatology (Oxford) 2013; 52: 1840-4.

[5] Stone JH, Merkel PA, Spiera R, et al. Rituximab versus cyclophosphamide for ANCA-associated vasculitis. N Engl J Med 2010; 363: 221-32.

[6] Jones RB, Tervaert JW, Hauser T, et al. Rituximab versus cyclophosphamide in ANCA-associated renal vasculitis. N Engl J Med 2010; 363: 211-20.

[7] Bykerk VP, Schieir O, Akhavan P, Hazlewood GS, Cheng CK, Bombardier C. Emerging issues in pharmacological management of rheumatoid arthritis: results of a national needs assessment survey identifying practice variations for the development of Canadian Rheumatology Association clinical practice recommendations. J Rheumatol 2012; 39: 1555-8.

[8] Kidney Disease: Improving Global Outcomes (KDIGO) Glomerulonephritis Work Group. KDIGO Clinical Practice Guideline for Glomerulonephritis. Kidney Int Suppl 2012; 139274.

[9] Roubaud-Baudron C, Pagnoux C, Meaux-Ruault $\mathrm{N}$, et al. Rituximab maintenance therapy for granulomatosis with polyangiitis and microscopic polyangiitis. J Rheumatol 2012; 39: 125-30.

[10] Cartin-Ceba R, Golbin JM, Keogh KA, et al. Rituximab for remission induction and maintenance in refractory granulomatosis with polyangiitis (Wegener's): ten-year experience at a single center. Arthritis Rheum 2012; 64: 3770-8.

[11] Smith RM, Jones RB, Guerry MJ, et al. Rituximab for remission maintenance in relapsing antineutrophil cytoplasmic antibodyassociated vasculitis. Arthritis Rheum 2012; 64: 3760-9.

[12] Guillevin L, Pagnoux C, Karras A, et al. Rituximab versus Azathioprine for Maintenance in ANCA-Associated Vasculitis. N Engl J Med 2014; 371: 1771-80. 